\title{
Câncer colorretal - resultados da avaliação patológica padronizada de 521 casos operados no Hospital das Clínicas da UFMG
}

\author{
Colorectal cancer - results of standardized pathologic evaluation of 521 patients \\ underwent surgery at Hospital das Clínicas, Federal University of Minas Gerais
}

\author{
LEONARDO MACIEL DA FONSECA ${ }^{1}$, LUCAS VIANA QUITES ${ }^{2}$, MÔNICA MARIA DEMAS ÁLVARES CABRAL ${ }^{2}$, \\ RODRIGO GOMES DA SILVA ${ }^{1}$, MAGDA MARIA PROFETA DA LUZ ${ }^{1}$, ANTÔNIO LACERDA FILHO' \\ ${ }^{1}$ Grupo de Coloproctologia e Intestino Delgado do Instituto Alfa de Gastroenterologia do Hospital das Clínicas da \\ Universidade Federal de Minas Gerais. ${ }^{2}$ Laboratório de Patologia Cirúrgica do Instituto Alfa de Gastroenterologia do \\ Hospital das Clínicas da Universidade Federal de Minas Gerais e Departamento de Anatomia Patológica e Medicina \\ Legal da Faculdade de Medicina da Universidade Federal de Minas Gerais.
}

FONSECA LM, QUITES LV, CABRAL MMDA, SILVA RG, LUZ MMP, FILHO AL. Câncer colorretal-resultados da avaliação patológica padronizada de 521 casos operados no Hospital das Clínicas da UFMG. Rev bras Coloproct, 2011;31(1): 17-25.

RESUMO: Introdução: A incidência e a mortalidade por câncer colorretal (CCR) têm apresentado, em todo mundo, uma tendência ao crescimento, em especial em países desenvolvidos e em áreas urbanas de países menos desenvolvidos. O estudo das características anatomopatológicas do tumor é importante para o estadiamento e a definição da terapêutica a ser empregada. O objetivo deste estudo é avaliar as características anatomopatológicas de peças cirúrgicas de ressecções colorretais por neoplasias no Hospital das Clínicas da Universidade Federal de Minas Gerais. Resultados: Foram avaliadas 521 peças cirúrgicas de ressecções colorretais. A idade média dos pacientes foi de $62,47( \pm 14,67)$ anos, sendo $302(58 \%)$ deles do sexo feminino. Os tumores do cólon esquerdo foram os mais comuns (340 [65,3\%]). Houve predominância de adenocarcinomas (457 [87,7\%]), úlcero-infiltrativos (176 [33,8\%]), moderadamente diferenciados (396 [76\%]), pT3 (316 [60,7\%]) e pN0 (213 [40,9\%]). O número médio de linfonodos dissecados por peça cirúrgica foi de 22,13 ( $(14,27)$. Conclusões: Os dados de nossa casuística de CCR não diferiram do que foi relatado na literatura. Em síntese, o CCR foi mais comum em mulheres do que em homens, acometeu com maior frequência o cólon esquerdo, e o tipo predominante foi o adenocarcinoma moderadamente diferenciado, pT3 e pNO.

Palavras-chave: neoplasias colorretais; cirurgia colorretal; estadiamento de neoplasias; histologia; patologia cirúrgica.

\section{INTRODUÇÃO}

A incidência e a mortalidade por câncer colorretal (CCR) têm apresentado, em todo mundo, uma tendência ao crescimento, em especial em países desenvolvidos e em áreas urbanas de países menos desenvolvidos ${ }^{1,2}$. No Brasil, assim como em outros países em desenvolvimento, com o aumento da expectativa de vida da população, as neoplasias vêm ganhando cada vez mais importância no perfil de morbidade e mortalidade ${ }^{3}$. Segundo estimativas do Ministério da Saúde/Instituto
Nacional do Câncer (INCA) são previstos, para o ano de 2010, 13.310 novos casos de câncer de cólon e reto em homens e 14.800 em mulheres ${ }^{4}$.

As características anatomopatológicas do tumor são importantes para o estadiamento e a definição da terapêutica a ser empregada ${ }^{5-7}$. O nível de infiltração da parede do órgão, assim como invasão de veias, acometimento de nervos ${ }^{8}$ e metástases linfonodais ${ }^{9}$ ou em órgãos à distância, está intimamente relacionado com pior prognóstico da doença ${ }^{6}$. O estadiamento convencional é feito pelas classificações de Dukes ${ }^{7}$ e

Conflito de interesse: nada a declarar.

Recebido em: 01/12/2010

Aprovado em: 14/04/2011 
Astler-Coller ${ }^{5}$ e $\mathrm{TNM}^{10}$, sendo a última cada vez mais utilizada.

O objetivo deste estudo foi avaliar as características anatomopatológicas de peças cirúrgicas de ressecções colorretais por neoplasias em uma instituição universitária, com serviços de coloproctologia e anatomia patológica dedicados, a partir do estudo dos laudos padronizados de CCR estabelecidos em 2001, quando tais características passaram a ser coletadas prospectivamente em banco de dados.

\section{MATERIAIS E MÉTODOS}

Foram avaliados os espécimes cirúrgicos de pacientes operados por CCR no Grupo de Coloproctologia e Intestino Delgado do Instituto Alfa de Gastroenterologia do Hospital das Clínicas da Universidade Federal de Minas Gerais (IAG/HC-UFMG), entre o período de janeiro de 2001 a abril de 2010. As peças cirúrgicas foram analisadas por patologistas especializados em patologia gastrointestinal do Laboratório de Patologia Cirúrgica do IAG/HC-UFMG e do Departamento de Anatomia Patológica e Medicina Legal da Faculdade de Medicina da UFMG.

Os dados foram levantados a partir das requisições dos exames e de laudos anatomopatológicos padronizados específicos para CCR implantados em $2001^{11}$. As variáveis estudadas foram sexo, idade dos pacientes, topografia da lesão, classificação macroscópica, dimensões do tumor, número de linfonodos totais e com metástases, classificação histológica e diferenciação tumoral, resposta linfocítica peritumoral, invasão venosa, linfática e neural e estadiamento $\mathrm{TNM}^{10}$.

A idade foi expressa em média e desvio-padrão (DP), e os pacientes foram divididos em oito grupos etários (até 30 anos, de 31 a 40 anos, 41 a 50 anos, 51 a 60 anos, 61 a 70 anos, 71 a 80 anos, 81 a 90 anos e maior que 91 anos). Quanto à topografia, os casos foram agrupados em tumores do cólon direito, englobando tumores desde o ceco até o cólon transverso, e do cólon esquerdo, da flexura esplênica ao reto. Tumores localizados na transição sigmoide/reto foram classificados como de retossigmoide. Os tumores foram classificados macroscopicamente como polipoide, séssil, em forma de placa, úlcero-infiltrativo, anular constritivo, difuso, ulcerado e outros. As dimensões do tumor mensuradas foram seu comprimento longitudinal, transversal e profundidade, expressas em centímetros, assim como o tamanho da peça cirúrgica. A classificação histológica e a diferenciação tumoral estão de acordo com a aquela preconizada pela Organização Mundial da Saúde (OMS) ${ }^{12}$. Por ser tratar de um estudo exclusivo de peças cirúrgicas, foram avaliadas apenas as características do tumor $(\mathrm{T})$ e o envolvimento linfonodal $(\mathrm{N})$ do estadiamento TNM.

Os resultados foram analisados com emprego do programa de computador Statistical Package for Social Sciences (SPSS) para Windows ${ }^{\circledR}$ versão 17.0 (SPSS, Chicago, Illinois). Variáveis contínuas foram expressas em média e DP, e os grupos comparados com teste U de Mann-Whitney. Variáveis categóricas foram comparadas com os testes qui-quadrado. Os valores de $\mathrm{p}<0,05$ foram considerados estatisticamente significativos.

\section{RESULTADOS}

Foram avaliadas 521 peças cirúrgicas de ressecções colorretais, de cujos laudos padronizados foram obtidas as características patológicas que compuseram o banco de dados estudado. A idade média dos pacientes foi de $62,47( \pm 14,67)$ anos, sendo 302 deles $(58 \%)$ do sexo feminino. Em relação aos grupos etários, 5 pacientes (1\%) tinham até 30 anos, $31(6 \%)$ de 31 a 40 anos, $79(15,2 \%)$ de 41 a 50 anos, $113(21,7 \%)$ de 51 a 60 anos, também $113(21,7 \%)$ de 61 a 70 anos, 122 $(23,4 \%)$ de 71 a 80 anos, $52(10,0 \%)$ de 81 a 90 anos e $6(1,2 \%)$ mais que 91 anos (Figura 1$)$.

Nesta casuística foram identificados 181 (34,7\%) casos de tumores de cólon direito. Dentre esses, 67 (12,9\%) encontravam-se no ceco, 62 (11,9\%) no cólon ascendente, $18(3,5 \%)$ na flexura hepática e 34 (6,5\%) no cólon transverso. Dos $340(65,3 \%)$ tumores localizados no cólon esquerdo, 11 (2,1\%) situavam-se em ângulo esplênico, 35 (6,7\%) em cólon descendente, $117(22,5 \%)$ no cólon sigmoide, 59 (11,3\%) no retossigmoide e $118(22,6 \%)$ no reto (Figura 2).

O tamanho médio das peças cirúrgicas foi de $30,35( \pm 21,19) \mathrm{cm}$. As dimensões médias longitudinal, transversal e de profundidade dos tumores foram $5,14( \pm 3,73) \mathrm{cm}, 4,38( \pm 3,88) \mathrm{cm} \mathrm{e} 2,20( \pm 2,89) \mathrm{cm}$, respectivamente.

A classificação histológica dos tumores foi a seguinte: 457 (87,7\%) eram adenocarcinomas, $3(0,6 \%)$ 
tumores de células em anel de sinete, $2(0,4 \%)$ tumores de pequenas células, $45(8,6 \%)$ tumores mucinosos, 1 $(0,2 \%)$ tumor basaloide, $3(0,6 \%)$ do tipo escamoso, $6(1,2 \%)$ adenoescamosos, $1(0,2 \%)$ tumor classificado como indiferenciado e $3(0,6 \%)$ peças em que não foi identificado tumor residual. $\mathrm{O}$ aspecto macroscópico dos tumores foi 130 (25\%) lesões polipoides, $47(9,0 \%)$ sésseis, $18(3,5 \%)$ em placa, $176(33,8 \%)$ úlcero-infiltrativos, $138(26,5 \%)$ anulares constritivos, $3(0,6 \%)$ com aspecto difuso, $1(0,2 \%)$ ulcerado e 8 $(1,5 \%)$ com outros aspectos macroscópicos. Do ponto de vista da diferenciação celular, esta série englobou três $(0,6 \%)$ tumores indiferenciados, 34 (6,5\%) pouco diferenciados, 396 (76\%) moderadamente diferenciados, $83(15,9 \%)$ bem diferenciados e $5(1,0 \%)$ em que esta classificação não se aplicava.

O número médio de linfonodos dissecados por peça cirúrgica foi de $22,13( \pm 14,27)$. O número médio de linfonodos com metástases foi de $2,57( \pm 5,34)$. Dos tumores avaliados, $130(25,0 \%)$ apresentaram invasão venosa, $267(51,2 \%)$ invasão linfática e $141(27,1 \%)$ invasão neural. Com relação à resposta linfocítica peritumoral, $163(31,3 \%)$ não apresentavam resposta, $264(50,7 \%)$ discreta resposta, $76(14,6 \%)$ resposta moderada e $18(3,5 \%)$ resposta acentuada.

A classificação T dos tumores avaliados foi a seguinte: 25 (4,8\%) pT1; 105 (20,2\%) pT2; $316(60,7 \%)$ pT3; 66 (12,7\%)pT4; $7(1,3 \%)$ T0 e dois $(0,4 \%)$ pTis (Figura 3). Já a classificação $\mathrm{N}$ foi a seguinte: $112(21,5 \%)$ pN1; 95 (18,2\%) pN2; 8 (1,5\%) pN3, 93 (17,9\%) pNx, $213(40,9 \%)$ pN0 (Figura 4). De acordo com a classificação Astler-Coller, 6 (1,2\%) foram classificados como A, 99 (19\%) como B1, 135 (25,9\%) como B2, 46 (8,8\%) como C1, 201 (38,6\%) como C2, 29 (5,6\%) como D e $5(1,0 \%)$ eram lesões não aplicáveis para essa classificação (Figura 5). E, de acordo com a classificação de Dukes, 99 (19,0\%) foram classificados como A, 155 (29,8\%) como B, 232 (44,5\%) como C, 29 (5,6\%) como D e $6(1,2 \%)$ não aplicáveis (Figura 6).

Os tumores tinham características semelhantes tanto em homens como em mulheres, sem diferença em relação à idade, localização, dimensões do tumor, diferenciação tumoral, invasão venosa, linfática e neural, classificação PT e pN, Astler-Coller e Dukes, número de linfonodos totais e com metástase (Tabela 1).

Os pacientes com tumores do cólon direito apresentaram idade maior que os pacientes com tumo-

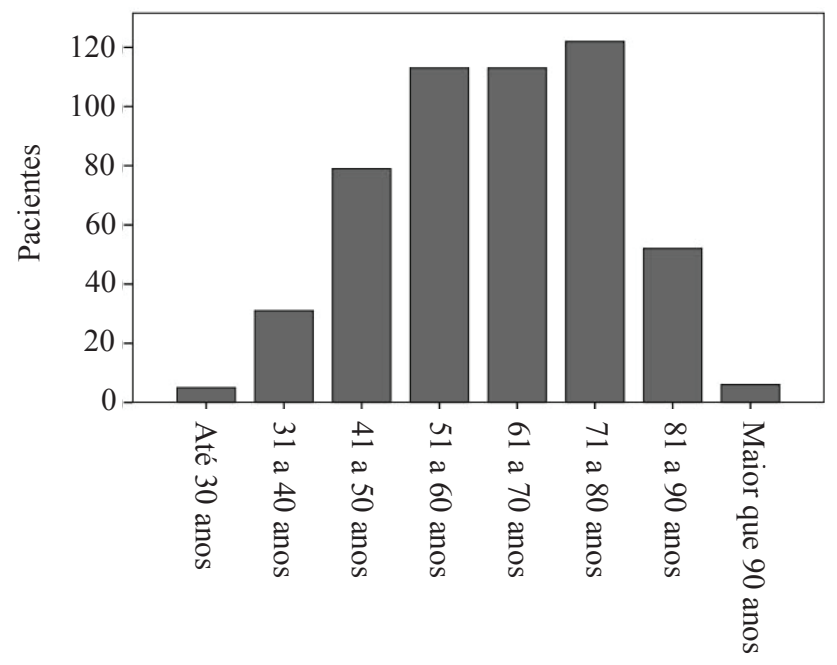

Figura 1. Número de pacientes com CCR por faixa etária.

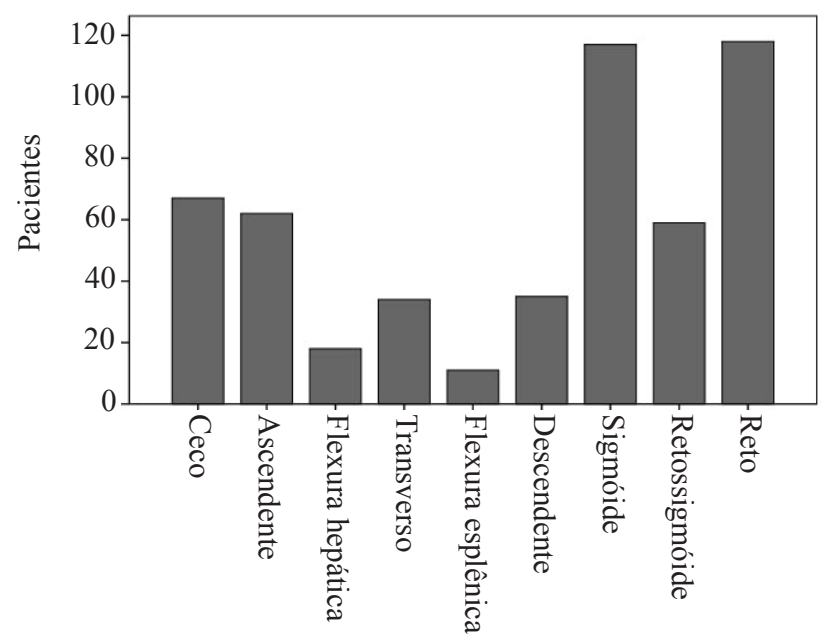

Figura 2. Número de tumores por localização.

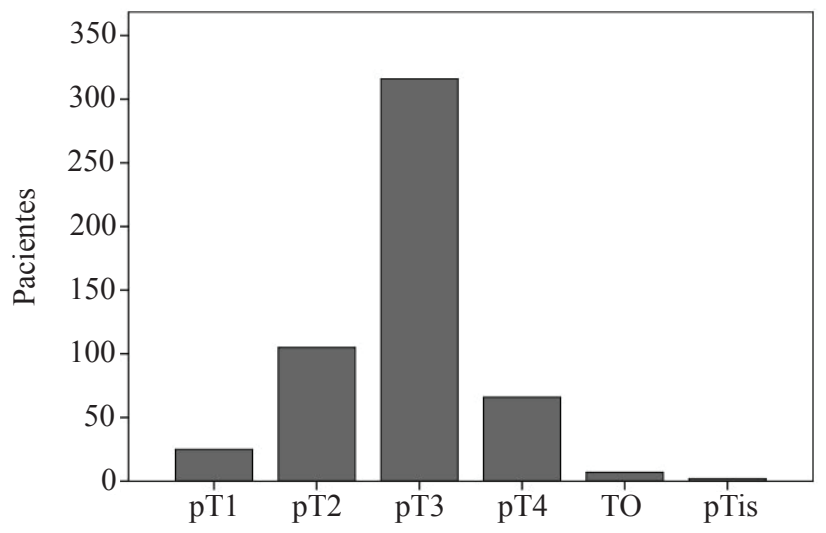

Figura 3. Classificação $p T$. 


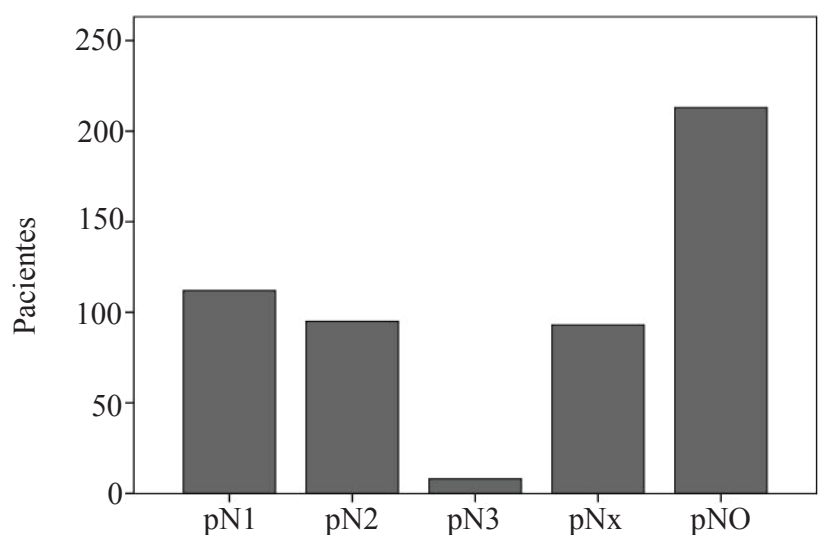

Figura 4. Classificação $p N$.

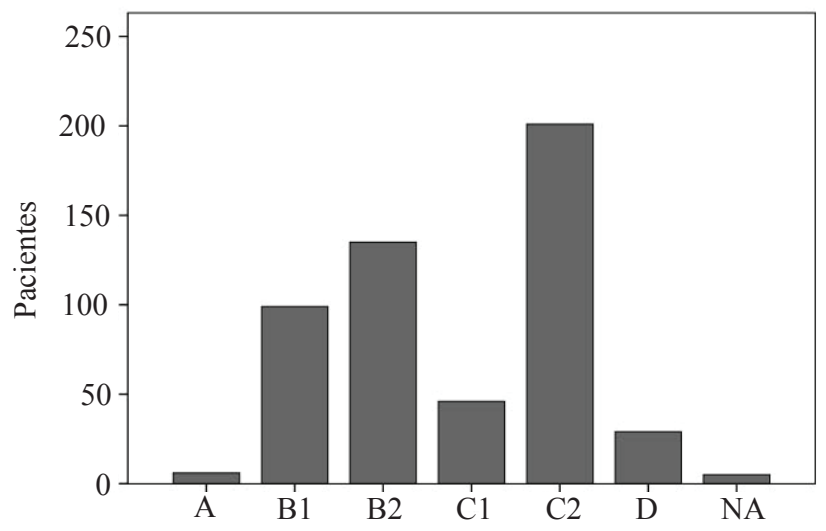

Figura 5. Classificação Astler-Coller.

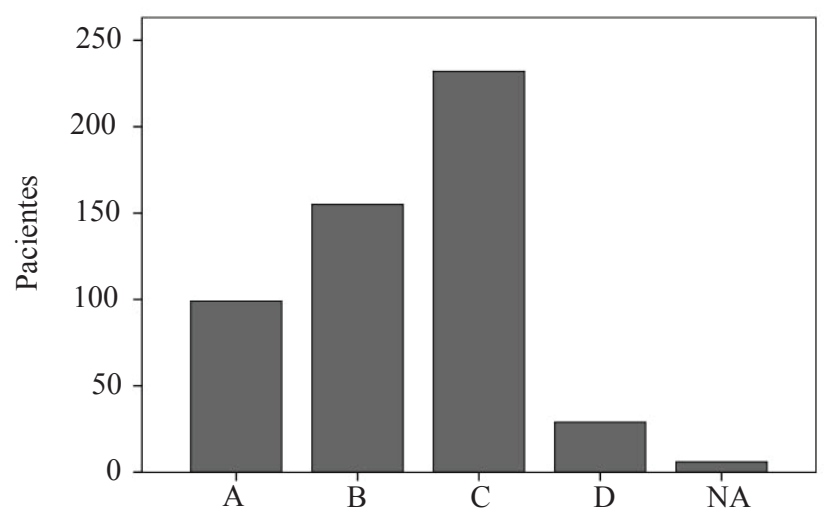

Figura 6. Classificação de Dukes.

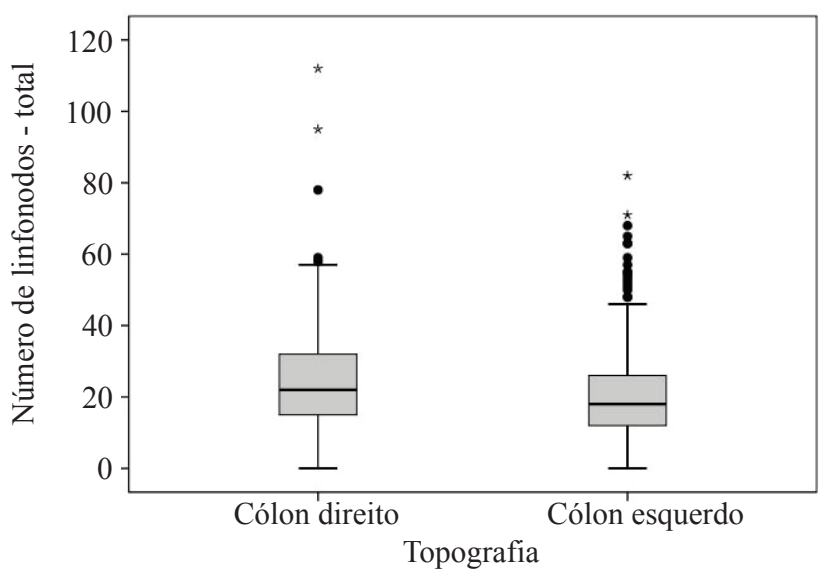

Figura 7. Comparação do número de linfonodos total com topografia do tumor ( $p=0,000$; Teste U de Mann-Whitney).

res do cólon esquerdo $(64,92[ \pm 15,10]$ versus 61,16 $[ \pm 14,28] ; p=0,004)$. Os tumores do cólon direito foram significativamente maiores que os do cólon esquerdo (longitudinal $[5,78\{ \pm 4,58\}$ versus 4,80 $\{ \pm 3,13\}, p=0,000]$; transversal $[5,17\{ \pm 5,14\}$ versus $3,96\{ \pm 2,93\}, \mathrm{p}=0,000]$; profundidade $[2,35\{ \pm 2,04\}$ versus $2,13\{ \pm 3,25\}, \mathrm{p}=0,000])$. Além disso, nas peças cirúrgicas do cólon direito foram obtidos mais linfonodos que nas do cólon esquerdo $(25,03[ \pm 15,78]$ versus 20,58 $[ \pm 13,16] ; p=0,000$ ) (Figura 7), sendo que o número de linfonodos metastáticos não apresentou diferença em relação à topografia $(2,56[ \pm 4,61]$ versus $2,58[ \pm 5,70] ; p=0,729)$. As outras variáveis avaliadas não apresentaram diferença estatisticamente significativa quanto às características do CCR em relação à localização (Tabela 2).

\section{DISCUSSÃO}

A análise das peças cirúrgicas de 521 pacientes operados por neoplasias de cólon em um único serviço mostrou prevalência do sexo feminino, perfil concordante com as estimativas previstas pelo INCA para o ano de $2010^{4}$, com outras séries nacionais ${ }^{13,14}$ e levantamentos norte-americanos ${ }^{15,16}$ e europeus ${ }^{17}$. A população mais afetada tinha entre 50 e 80 anos, sendo a idade média de 63 anos, semelhante à encontrada na literatu$\mathrm{ra}^{2}, 18,19$, mostrando prevalência da doença na população de faixa etária mais alta. Por outro lado, deve ser ressaltado, na nossa casuística, o elevado número de 
Tabela 1. Comparação entre os gêneros e características histopatológicas dos tumores.

\begin{tabular}{|c|c|c|c|c|}
\hline & & Masculino & Feminino & $\mathbf{p}$ \\
\hline Idade (anos) & Média (DP) & $61,95( \pm 14,00)$ & $62,84( \pm 15,15)$ & $0,465^{*}$ \\
\hline \multirow[t]{2}{*}{ Localização } & Cólon direito & 75 & 106 & $0,840^{\#}$ \\
\hline & Cólon esquerdo & 144 & 196 & \\
\hline \multicolumn{5}{|l|}{ Dimensões do tumor (cm) } \\
\hline Longitudinal & Média (DP) & $5,62( \pm 5,13)$ & $4,79( \pm 2,16)$ & $0,069^{*}$ \\
\hline Transversal & Média (DP) & $4,64( \pm 3,74)$ & $4,19( \pm 3,98)$ & $0,050^{*}$ \\
\hline Profundidade & Média (DP) & $2,29( \pm 3,07)$ & $2,14( \pm 2,76)$ & $0,603^{*}$ \\
\hline \multirow[t]{5}{*}{ Diferenciação tumoral } & Indiferenciado & 2 & 1 & $0,289^{\ddagger}$ \\
\hline & $\begin{array}{c}\text { Pouco } \\
\text { diferenciado }\end{array}$ & 19 & 15 & \\
\hline & $\begin{array}{l}\text { Moderadamente } \\
\text { diferenciado }\end{array}$ & 162 & 234 & \\
\hline & Bem diferenciado & 33 & 50 & \\
\hline & Não se aplica & 3 & 2 & \\
\hline \multirow{2}{*}{ Invasão venosa } & não & 162 & 229 & $0,629^{*}$ \\
\hline & $\operatorname{sim}$ & 57 & 73 & \\
\hline \multirow[t]{2}{*}{ Invasão linfática } & não & 111 & 143 & $0,452^{\#}$ \\
\hline & $\operatorname{sim}$ & 108 & 159 & \\
\hline \multirow[t]{2}{*}{ Invasão neural } & não & 158 & 222 & $0,729^{\#}$ \\
\hline & $\operatorname{sim}$ & 61 & 80 & \\
\hline \multirow[t]{6}{*}{$\mathrm{pT}$} & pT1 & 12 & 13 & $0,836^{\ddagger}$ \\
\hline & pT2 & 41 & 64 & \\
\hline & pT3 & 136 & 180 & \\
\hline & pT4 & 27 & 39 & \\
\hline & T0 & 2 & 5 & \\
\hline & pTis & 1 & 1 & \\
\hline \multirow[t]{5}{*}{$\mathrm{pN}$} & $\mathrm{pN} 1$ & 40 & 72 & 0,107 \\
\hline & $\mathrm{pN} 2$ & 40 & 55 & \\
\hline & $\mathrm{pN} 3$ & 3 & 5 & \\
\hline & $\mathrm{pNx}$ & 38 & 55 & \\
\hline & pNo & 98 & 115 & \\
\hline \multirow[t]{7}{*}{ Astler-Coller } & A & 1 & 5 & $0,441^{\ddagger}$ \\
\hline & B1 & 44 & 55 & \\
\hline & B2 & 64 & 71 & \\
\hline & $\mathrm{C} 1$ & 16 & 30 & \\
\hline & $\mathrm{C} 2$ & 79 & 122 & \\
\hline & $\mathrm{D}$ & 13 & 16 & \\
\hline & NA & 2 & 3 & \\
\hline \multirow[t]{5}{*}{ Dukes } & A & 42 & 57 & $0,631^{\ddagger}$ \\
\hline & $\mathrm{B}$ & 72 & 83 & \\
\hline & $\mathrm{C}$ & 88 & 144 & \\
\hline & $\mathrm{D}$ & 14 & 15 & \\
\hline & NA & 3 & 3 & \\
\hline \multirow{2}{*}{$\begin{array}{l}\text { Número de } \\
\text { linfonodos }\end{array}$} & Média (DP) & $22,45( \pm 13,77)$ & $21,89( \pm 14,64)$ & $0,562^{*}$ \\
\hline & Média (DP) & $2,79( \pm 6,33)$ & $2,42( \pm 4,50)$ & $0,707^{*}$ \\
\hline
\end{tabular}

* Teste U de Mann-Whitney; \# Teste qui-quadrado; $\neq$ Teste qui-quadrado (associação linear) 
Tabela 2. Comparação entre cólons direito e esquerdo e características histopatológicas dos tumores.

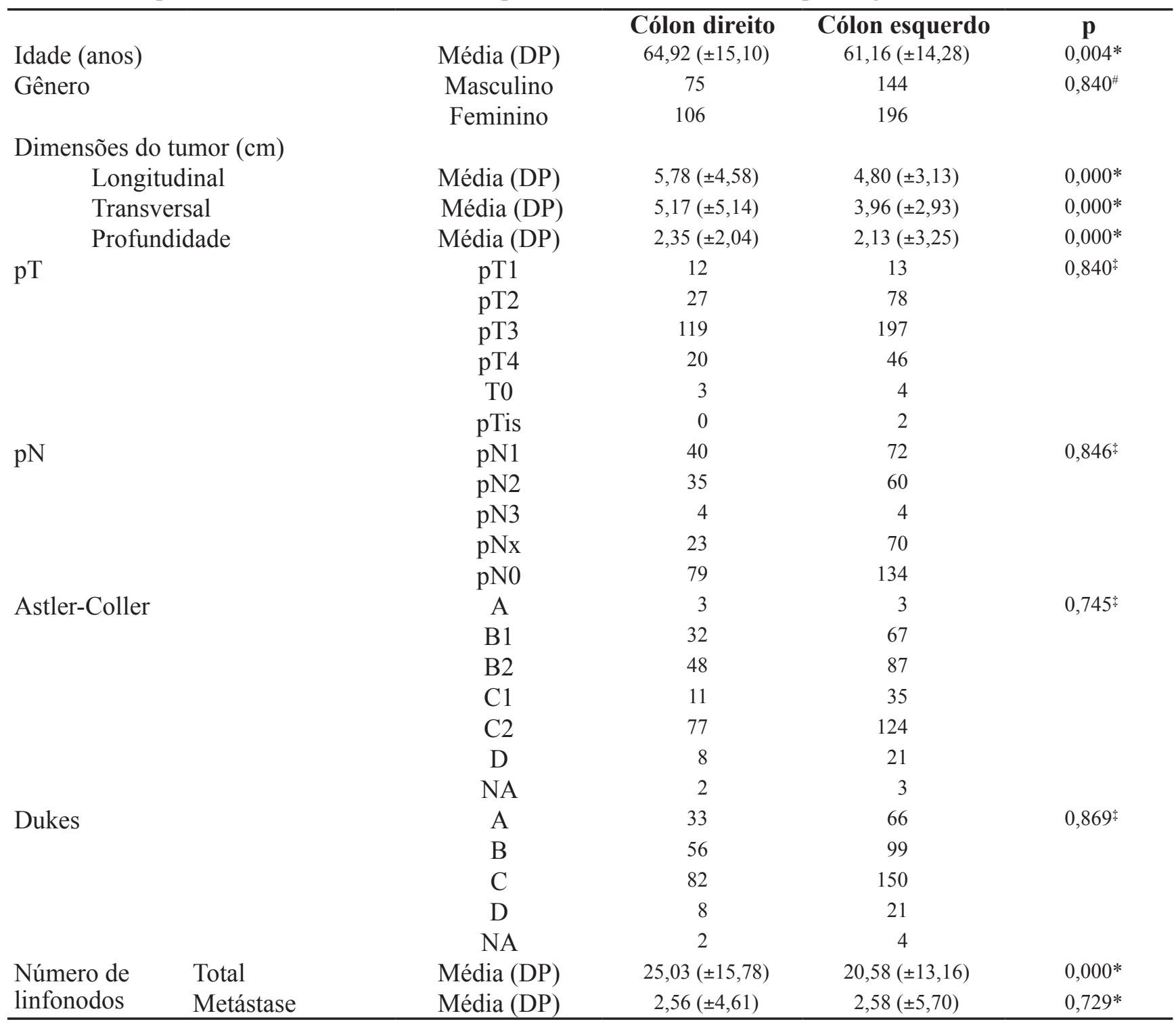

* Teste U de Mann-Whitney; \# Teste qui-quadrado; $\neq$ Teste qui-quadrado (associação linear)

pacientes com menos de 40 anos, que representavam $7,0 \%$ da amostra (36 pacientes). Outra série nacional que avaliou pacientes com CCR com idade inferior a 40 anos, durante um período de 10 anos, relatou apenas 11 casos de CCR nessa faixa etária ${ }^{20}$. Destaca-se ainda o significante número de linfonodos dissecados por peça cirúrgica, consideravelmente maior que em outros estudos $^{21-23}$. Não foram notadas diferenças significativas em relação aos dados anatomopatológicos estudados entre homens e mulheres. Nesta série destacou-se também, em relação à topografia, que tumo- res localizados à direita apresentaram maior volume, característica já bem estabelecida, e que nessas peças cirúrgicas foram obtidos mais linfonodos que em peças do cólon esquerdo.

Nos dias atuais, o CCR destaca-se mundialmente como uma neoplasia de importante significado, sendo que, em 2008, a Agência Internacional para a Pesquisa do Câncer, vinculada à Organização Mundial da Saúde, estimou que ocorreriam 12,4 milhões de casos novos e 7,6 milhões de óbitos por câncer no mundo 3 . Dentre esses, o CCR foi a terceira causa mais comum, 
com 1,15 milhão de casos, atrás apenas das neoplasias pulmonares e de mama, sendo que cerca de um terço dos casos ocorreriam em países em desenvolvimento $^{3}$. Estudo brasileiro com o objetivo de avaliar a mortalidade por CCR entre os anos de 1980 e 1997 demonstrou aumento crescente dessa taxa ${ }^{24}$. Torna-se, portanto, de grande importância o estudo da apresentação dessa neoplasia em nosso meio, através da avaliação de grandes séries de casos. Após pesquisa da literatura nacional, não foi encontrada casuística publicada maior do que a presente série com o objetivo de analisar características patológicas de neoplasias colorretais $^{13,25,26}$

Outras casuísticas, como a de Cruz et al., analisaram as características de 490 pacientes com diagnóstico de $\mathrm{CCR}^{25}$. Nesse estudo, características como prevalência dos tumores à esquerda e idade de acometimento foram semelhantes a esta série. Nossos resultados são divergentes se comparados a esse estudo em relação à prevalência de tumores por gênero e classificação de Dukes. Enquanto em nosso estudo o sexo feminino foi mais acometido e predominou a classificação C de Dukes, no outro estudo houve maior prevalência do sexo masculino e foram observados mais pacientes com classificações A e B de Dukes. Isto talvez se deva ao fato de o estudo de Cruz et al. ${ }^{25}$ ter sido feito, em grande parte, com pacientes operados na clínica privada do autor principal, na qual os pacientes de maior nível socioeconômico teriam maior acesso a um diagnóstico mais precoce da doença, ao contrário do observado nos pacientes da presente série, pertencentes a classes socioeconômicas menos favorecidas. Por outro lado, outras séries de Santa Catarina, com 251 casos de CCR, e de São Paulo, com 51 casos, apresentaram resultados semelhantes aos aqui relatados ${ }^{13,26}$.

Destaca-se nesta série o elevado número de linfonodos dissecados por peças cirúrgicas. É sabido que, quanto maior o número de linfonodos avaliados, maior a chance de um diagnóstico mais preciso e, consequentemente, de se determinar a necessidade da utilização de terapia adjuvante e inferir um prognóstico para cada caso específico. Esse elevado número, superior a média relatada pela literatura ${ }^{21-23}$, é atribuído a uma ressecção cirúrgica oncologicamente adequada e, principalmente, a um maior empenho no estudo do espécime cirúrgico, praticado por especialista dedicado à patologia digestiva $\mathrm{e}$, sobretudo, colorretal $^{27}$. Além disso, a utilização de solução clareadora de linfonodos tem papel fundamental na obtenção de um maior número de linfonodos da peça cirúrgica. Comparando peças de ressecções colorretais em que a obtenção de linfonodos foi realizada com peças sem a utilização desta solução, as primeiras apresentam número significativamente maior ${ }^{28}$.

A literatura tem apontado para um aumento da incidência de tumores no cólon direito nos últimos $\operatorname{anos}^{2,17,29,30}$. Na nossa série, notou-se um predomínio de tumores no cólon esquerdo, principalmente sigmoide, retossigmoide e reto. Essa predominância pode corresponder a um viés de seleção, pois o HC-UFMG é um centro de referência no estado de Minas Gerais, para onde são encaminhados os casos de maior complexidade, ou seja, pacientes com tumores dessas localizações, sobretudo do reto, nos quais as operações necessariamente devem ser realizadas por cirurgiões especialistas.

O CCR apresentou variação de volume relacionada com a sua localização. No presente estudo houve diferença significativa entre todas as dimensões dos tumores do cólon direito (maiores) e esquerdo (menores). Essa diferença não foi acompanhada de piora dos critérios histopatológicos de prognóstico ou do estadiamento.

Observamos que em apenas 5,2\% dos pacientes o tumor infiltrava até a submucosa. De acordo com a classificação de Dukes ${ }^{7}$, apenas $19 \%$ dos pacientes apresentavam a doença em estágio inicial. Os $81 \%$ restantes tinham doença em estágio avançado, sendo a maioria (44,5\%) em estágio $C$, isto é, com doença metastática para linfonodos regionais, o que representa prognóstico significativamente pior para esses pacientes $^{5,7}$.

A invasão de nervos foi encontrada em $27,1 \%$ dos casos, de veias em $25 \%$ e de linfáticos em $51,2 \%$. Essas características são associadas a pior prognóstico ${ }^{18}$. A frequência de invasão dessas estruturas foi semelhante ao que se encontra na literatura ${ }^{18}$, entretanto, há que se salientar que o relato desses parâmetros histopatológicos não é estudado em número expressivo de casos em outras instituições, sobretudo não universitárias ${ }^{31}$.

Os dados de nossa casuística de CCR não diferiram do que foi relatado na literatura. Em síntese, o CCR foi mais comum em mulheres do que em homens, acometeu com maior frequência o cólon es- 
querdo, e o tipo predominante foi o adenocarcinoma moderadamente diferenciado, pT3 e pN0. Não se observou diferença quanto ao estadiamento em relação a sexo ou localização do tumor. Em cerca de $7 \%$ dos casos os pacientes tinham menos de 40 anos, sendo candidatos a testes genéticos para determinação do caráter hereditário da doença. No nosso meio, o CCR foi uma doença diagnosticada em fases avançadas, diminuindo acentuadamente a possibilidade de cura. Tal situação provavelmente reflete a desinformação com relação à doença, o que, seguramente, retarda a procura do paciente sintomático por assistência médica. Além disso, o sistema de saúde pública nacional ainda apresenta importantes falhas, no que diz respeito à disponibilização de meios diagnósticos à população carente, tais como o exame proctológico e a colonoscopia. Os achados encontrados no presente estudo, realizado em instituição pública de referência, podem não corresponder a casuísticas de CCR operados em instituições privadas nacionais, cujos dados não estão disponíveis na literatura. $\mathrm{O}$ panorama encontrado no presente estudo deve ensejar importantes mudanças nas políticas públicas de esclarecimento da população sobre a doença, além do desenvolvimento de estratégias de prevenção e diagnóstico precoce do CCR no Brasil, uma vez que os dados compilados pelo INCA não costumam especificar os diversos estágios em que a doença é diagnosticada.

\footnotetext{
ABSTRACT: Introduction: The incidence and mortality of colorectal cancer (CRC) have shown, worldwide, an upward trend, particularly in developed countries and urban areas of developing countries. The study of tumor's pathological characteristics is important for staging and the definition of the proper therapy to be used. The aim of this study is to evaluate the pathologic features of surgical specimens of colorectal resections due CRC at Hospital das Clínicas, Universidade Federal de Minas Gerais. Results: We evaluated 521 surgical specimens of colorectal resections. The mean age of patients was $62.47( \pm 14.67)$ years, $302(58 \%)$ were female. Tumors of the left colon were more common $(340[65.3 \%])$. There was a predominance of adenocarcinoma (457 [87.7\%]), ulcero-infiltrative (176 [33.8\%]), moderately differentiated (396 [76\%]), pT3 (316 [60.7\%]) and pN0 (213 [40.9\%]). The average number of dissected lymphonodes per surgical specimen was 22.13 ( \pm 14.27). Conclusions: Data from our series of CRC did not differ from what was reported in the literature. In summary, the CRC was more common in women than in men, affecting most frequently the left colon, and the predominant type was moderately differentiated adenocarcinoma, pT3 and pNO.
}

Keywords: colorectal neoplasms; colorectal surgery; neoplasm staging; histology; pathology; surgical.

\section{REFERÊNCIAS}

1. Garfinkel L, Mushinski M. U.S. cancer incidence, mortality and survival: 1973-1996. Stat Bull Metrop Insur Co. 1999;80(3):23-32.

2. Ponz de Leon M, Marino M, Benatti P, Rossi G, Menigatti M, Pedroni M, et al. Trend of incidence, subsite distribution and staging of colorectal neoplasms in the 15-year experience of a specialised cancer registry. Ann Oncol 2004;15(6):940-6.

3. Word Health Organization/International Agency for Research on cancer. World Cancer Report 2008. Lyon; 2009.

4. Brasil - Ministério da Saúde, Instituto Nacional do Câncer. Estimativa 2010: incidência de câncer no Brasil/Instituto Nacional de Câncer. Rio de Janeiro; 2009.

5. Astler VB, Coller FA. The prognostic significance of direct extension of carcinoma of the colon and rectum. Ann Surg 1954;139(6):846-52.

6. Bosman FT. Prognostic value of pathological characteristics of colorectal cancer. Eur J Cancer 1995;31A(7-8):1216-21.

7. Dukes CE. The classification of cancer of the rectum. J Pathol Bact 1932; 35: 323-32.

8. Phillips RK, Hittinger R, Blesovsky L, Fry JS, Fielding LP.
Large bowel cancer: surgical pathology and its relationship to survival. Br J Surg 1984;71(8):604-10.

9. Newland RC, Dent OF, Lyttle MN, Chapuis PH, Bokey EL. Pathologic determinants of survival associated with colorectal cancer with lymph node metastases. A multivariate analysis of 579 patients. Cancer 1994;73(8):2076-82.

10. Sobin LH, Wittekind CH. Colon and rectum. In: Sobin, LH, Wittekind, CH. TNM classification of malignant tumors. 6. ed. New York: Willy-Liss; 2002. p. 72-6.

11. Salles PGO, Nogueira AMMF. Câncer colorretal: preparo e análise de peças cirúrgicas. In: Castro, LP. Tópicos em gastroenterologia 11: avanços em coloproctologia. 1. ed. Rio de Janeiro: Editora Médica e Científica Ltda.; 2001. p. 167-74.

12. Harmilton SR. et al. Tumours of the colon and rectum. In: Harmilton SR, Altonen LA. World Healt Organization Classification of the Tumors. Pathology \& Genetics. Tumors or the digestive system. Lyon: IARC Press; 2000. p. 103-42.

13. Pinho MSL, Ferreira LC, Brigo MJK, Pereira Filho A, Wengerkievicz A, Ponath A, et al . Incidência do câncer colorretal na regional de saúde de Joinville (SC). Rev Bras Coloproct 2003;23(2):73-76. 
14. Reis RS, Santos MO, Bloch KV. Colorectal cancer in Porto Alegre and Fortaleza, Brazil: incidence trends and distribution pattern from 1990 to 1999. Cad. Saúde Pública, Rio de Janeiro. 2009;25(5):1046-1053.

15. Edwards BK, Ward E, Kohler BA, Eheman C, Zauber AG, Anderson RN, et al. Annual report to the nation on the status of cancer, 1975-2006, featuring colorectal cancer trends and impact of interventions (risk factors, screening, and treatment) to reduce future rates. Cancer 2010;116(3):544-73.

16. Matanoski G, Tao XG, Almon L, Adade AA, Davies-Cole JO. Demographics and tumor characteristics of colorectal cancers in the United States, 1998-2001. Cancer 2006;107(5 Suppl):1112-20.

17. Larsen IK, Bray F. Trends in colorectal cancer incidence in Norway 1962-2006: an interpretation of the temporal patterns by anatomic subsite. Int J Cancer 2010;126(3):721-32.

18. Duarte AP, Bromberg SH, Barreto E, Cappellano G, Godoy AC. Importância da invasão neural e linfática no prognóstico do adenocarcinoma colorretal. Rev Assoc Med Bras 2004; 50(1):21-6.

19. Koyama Y, Kotake K. Overview of colorectal cancer in Japan: report from the Registry of the Japanese Society for Cancer of the Colon and Rectum. Dis Colon Rectum 1997; 40(10Suppl):S2-9.

20. Carneiro Neto JD, Barreto JBP, Freitas NS, Queiroz MA. Câncer colorretal: características clínicas e anatomopatológicas em pacientes com idade inferior a 40 anos. Rev bras Coloproct 2006; 26(4):430-435.

21. Goldstein NS. Lymph node recoveries from 2427 pT3 colorectal resection specimens spanning 45 years: recommendations for a minimum number of recovered lymph nodes based on predictive probabilities. Am J Surg Pathol 2002;26(2):179-89.

22. Scott KW, Grace RH. Detection of lymph node metastases in colorectal carcinoma before and after fat clearance. Br J Surg 1989;76(11):1165-7.

23. Cserni G. The influence of nodal size on the staging of colorectal carcinomas. J Clin Pathol 2002;55(5):386-90.
24. das Neves FJ, Mattos IE, Koifman RJ. [Colon and rectal cancer mortality in Brazilian capitals, 1980-1997]. Arq Gastroenterol 2005;42(1):63-70.

25. Cruz GMG, Santana JL, Santana SKAA, Constantino JRM, Chamone BC, Ferreira RMRS, et al. Câncer colônico epidemiologia, diagnóstico, estadiamento e gradação tumoral de 490 pacientes. Rev bras Coloproct 2007;27(2):139-153.

26. Anderi JR E, Haddad MA, Agostinho AG, Dalaneze MC. Experiência de 14 anos no tratamento do câncer colorretal. Rev bras Coloproct 2001;21(3):148-52.

27. Pereira Jr T, Torres RAB, Nogueira AMMF. Acometimento metastático linfonodal no câncer colorretal. Arq Gastroenterol 2006;43(2):89-93.

28. Araújo SA, Cabral MMDA, Lacerda FA, Horta JGA, Luz MMP, Silva RG. Impacto do uso da solução reveladora de linfonodos no estadiamento do câncer colorretal. Rev Bras Coloproct 2009;29(3):279-86.

29. Troisi RJ, Freedman AN, Devesa SS. Incidence of colorectal carcinoma in the U.S.: an update of trends by gender, race, age, subsite, and stage, 1975-1994. Cancer 1999;85(8):1670-6.

30. Gervaz P, Bouzourene H, Cerottini JP, Chaubert P, Benhattar J, Secic M, et al. Dukes B colorectal cancer: distinct genetic categories and clinical outcome based on proximal or distal tumor location. Dis Colon Rectum 2001;44(3):364-72.

31. Silva RG, Cabral MD, Garcia P, Neiva A, Werneck BJ, Lacerda-Filho A. Número de linfonodos obtidos em peças cirúrgicas de câncer colorretal - o patologista faz a diferença. $57^{\circ}$ Congresso Brasileiro de Coloproctologia; 2008; Gramado/ RS. Rev bras Coloproct. 2008; 28:33.

\section{Endereço para correspondência:}

\section{ANTÔNIO LACERDA FILHO}

Instituto Alfa de Gastroenterologia

Hospital das Clínicas da UFMG - $2^{\circ}$ andar

Avenida Alfredo Balena, 110 - Bairro Santa Efigênia

CEP: 30130-100 - Belo Horizonte (MG), Brasil

E-mail: alacerdafilho@gmail.com 